\title{
An abstract drawing from the 73,000-year-old levels at Blombos Cave, South Africa
}

Christopher S. Henshilwood ${ }^{1,2}$, Francesco d'errico ${ }^{1,3}$, Karen L. van Niekerk $^{1}$, Laure Dayet ${ }^{3,4}$, Alain Queffelec $^{3} \&$ Luca Pollarolo ${ }^{5,6}$

\begin{abstract}
${ }^{1}$ SFF Centre for Early Sapiens Behaviour (SapienCE), University of Bergen, Bergen, Norway.
${ }^{2}$ Evolutionary Studies Institute, University of the Witwatersrand, Johannesburg, South Africa.

${ }^{3}$ CNRS UMR 5199, University of Bordeaux, Bordeaux, France.

${ }^{4}$ Laboratoire TRACES UMR 5608, Université Toulouse Jean Jaures, Toulouse, France.

${ }^{5}$ Unité d'Anthropologie/Laboratoire Archéologie et Peuplement de l'Afrique, Geneva, Switzerland.

${ }^{6}$ School of Geography, Archaeology and Environmental Studies, University of the Witwatersrand, Johannesburg, South Africa.
\end{abstract}

Abstract and depictive representations produced by drawing - known from Europe, Africa and Southeast Asia after 40,000 years ago-are a prime indicator of modern cognition and behaviour ${ }^{1}$. Here we report a cross-hatched pattern drawn with an ochre crayon on a ground silcrete flake recovered from approximately 73,000-year-old Middle Stone Age levels at Blombos Cave, South Africa. Our microscopic and chemical analyses of the pattern confirm that red ochre pigment was intentionally applied to the flake with an ochre crayon. The object comes from a level associated with stone tools of the Still Bay techno-complex that has previously yielded shell beads, cross-hatched engravings on ochre pieces and a variety of innovative technologies ${ }^{2-5}$. This notable discovery predates the earliest previously known abstract and figurative drawings by at least 30,000 years. This drawing demonstrates the ability of early Homo sapiens in southern Africa to produce graphic designs on various media using different techniques.

Blombos Cave (BBC) is situated on the southern Cape coast, about $300 \mathrm{~km}$ east of Cape Town $\left(34^{\circ}\right.$ $\left.24^{\prime} 51^{\prime \prime} \mathrm{S}, 21^{\circ} 13^{\prime} 19^{\prime \prime} \mathrm{E}\right)$. Excavations commenced in 1991 and are on-going. The site contains wellstratified Middle Stone Age (MSA) deposits dating to between 100 and 72 thousand years ago (ka)6, topped by a layer of sterile aeolian dune sand (dated to $70 \mathrm{ka}$ ) and Later Stone Age layers dated to $2 \mathrm{ka}$ (Fig. 1). The MSA sequence consists of four phases, of which the upper two-'BBC M1' and 'BBC M2 upper'-are associated with the Still Bay techno-complex, dated to about 77-73 ka7. These phases contain bifacial foliate points, of which $12 \%$ were heated before final shaping using pressure flaking 5,8 . Other cultural markers of the Still Bay from these layers include bone awls and spear points4, possible engravings of parallel and joining lines on bone4,9, beads made from Nassarius kraussianus shells (67 recovered thus far, some of which are stained with ochre3,10) and pieces of ochre engraved with geometric patterns, eight of which have been recovered thus far-of which two display distinct crosshatched designs2,11. The 'M2 lower' phase (dated to about $85-82 \mathrm{ka}$ ) is a low-intensity occupational horizon with no Still Bay type artefacts or engraved ochre. The M3 phase (dated to about 101-94 ka) contains abundant natural and used ochre, as well as ten pieces of ochre engraved with geometric designs that include three crosshatched motifs11. In addition, an in situ toolkit consisting of ochre, heated seal 
bone, charcoal and associated processing materials—used to create a liquid pigmented compound stored in abalone shells-was recovered from a layer of this phase that dates to about 100 ka6.

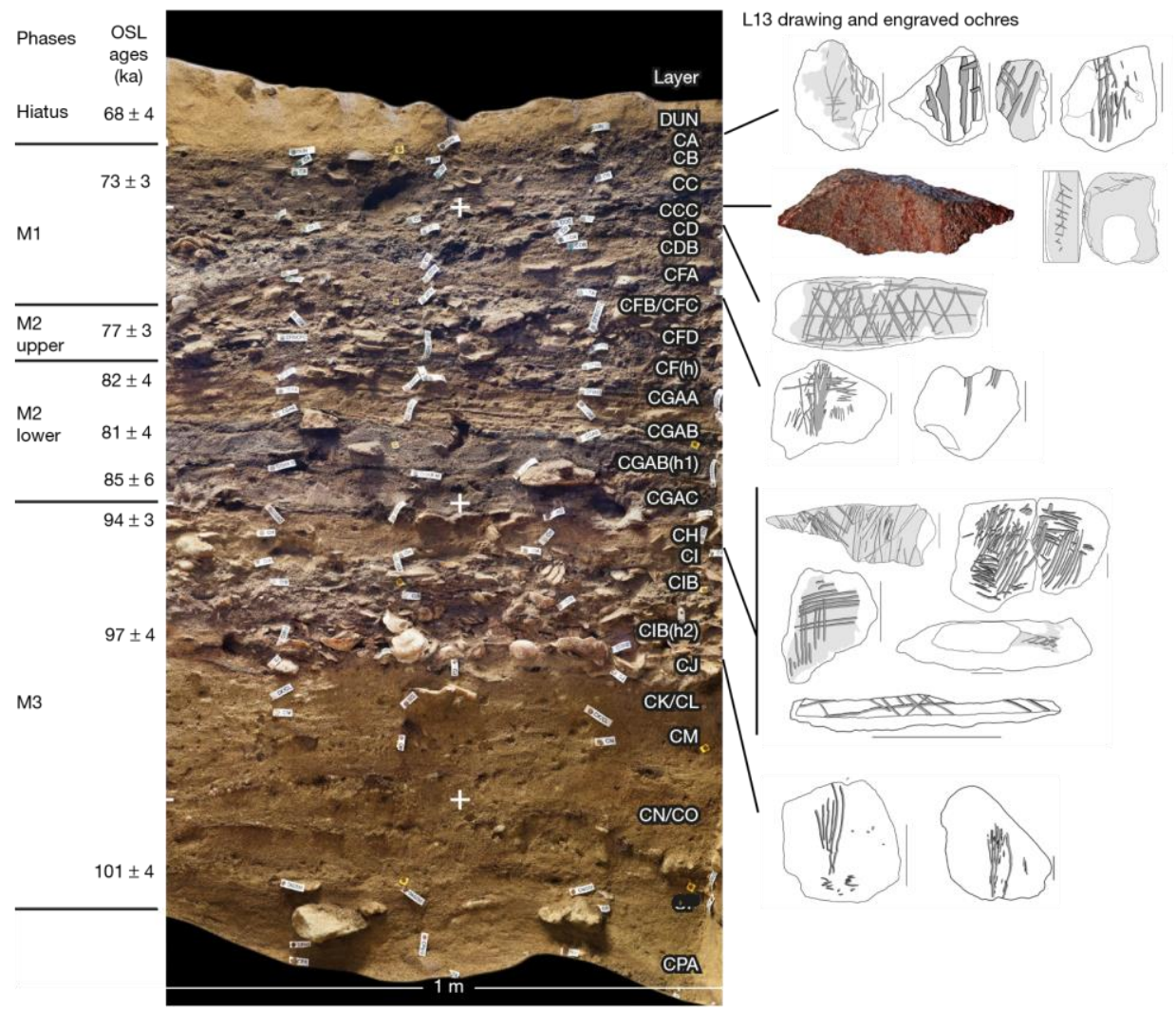

Fig. 1. Stratigraphy of the south section of Blombos Cave. Left, phases and optically stimulated luminescence dates for the Middle Stone Age levels at Blombos Cave. Centre, labels for individual layers superimposed on section. Right, layers from which the L13 silcrete flake with ochre drawings and previously described engraved ochre pieces were recovered. Scale bars, $1 \mathrm{~cm}$.

The silcrete flake with a cross-hatched pattern described here was excavated in 2011 and comes from the M1 phase (layer CCC, square G7b). The objects recovered from this layer were gently washed with running tap water in the field and the laboratory, and dried at air temperature. The pattern on the piece was noticed during analysis of the lithic debitage. The piece was numbered L13 in the laboratory (Fig. 2, Supplementary Data and Supplementary Video). The flake is coarse-grained silcrete (length 38.6 $\mathrm{mm}$, width $12.8 \mathrm{~mm}$ and height $15.4 \mathrm{~mm}$ ). The pattern is on a slightly concave smooth face-the flake's striking platform - and extends to a flake scar on the same surface. It consists of a set of six straight subparallel lines (Fig. 3, lines 1-6) crossed obliquely by three slightly curved lines (lines 7-9). Line 6 partially overlaps the edge of the flake scar, which suggests it was made after the flake became detached. All the lines are discontinuous and one (line 5) is wider and better defined than the others. Microscopic and chemical analyses confirm that the lines on L13 are composed of haematite-rich powder, commonly called ochre (Extended Data Fig. 1, Supplementary Information and Supplementary Table 2). They differ markedly in colour and composition from the silcrete and the orange calcite patches present on the same surface. The abrupt termination of all lines on the fragment edges indicates that the pattern originally extended over a larger surface (Fig. 3b) - the pattern was probably more complex and structured in its entirety than in this truncated form. 


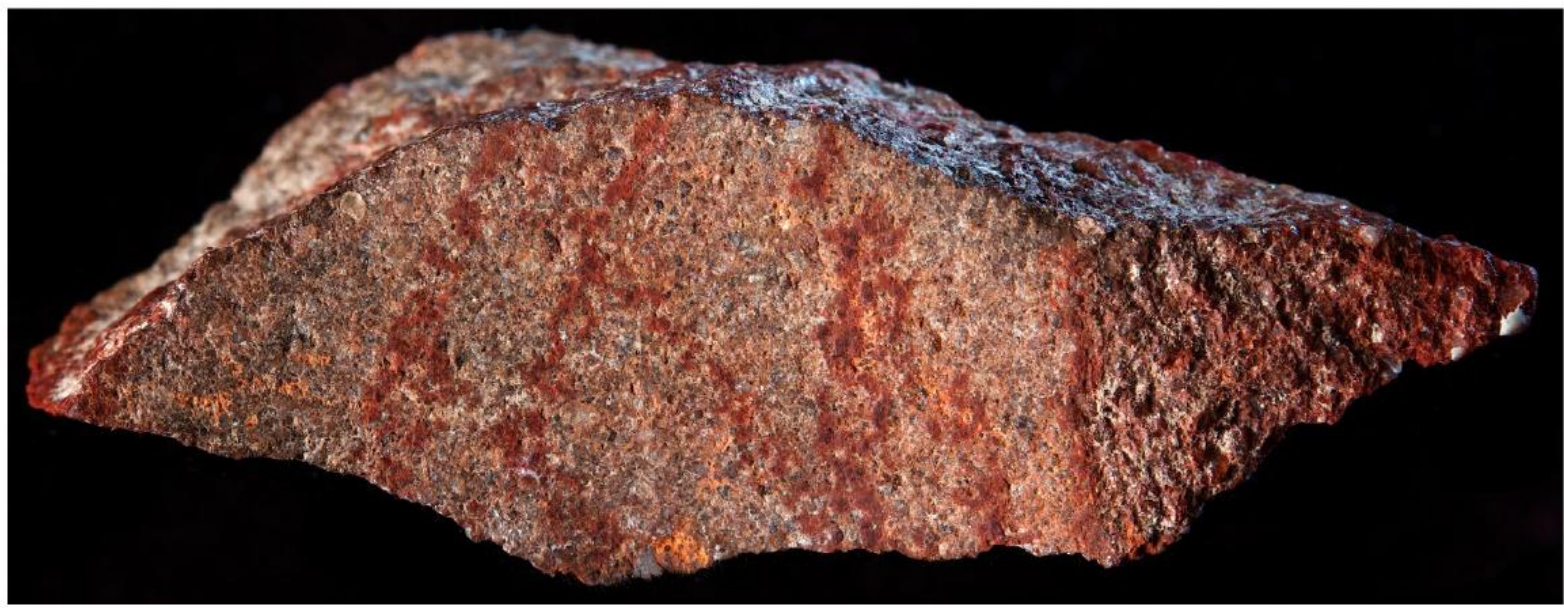

Fig. 2. Image of the Blombos Cave silcrete flake L13 displaying the drawn lines that form a cross-hatched pattern. Image credit, C. Foster.
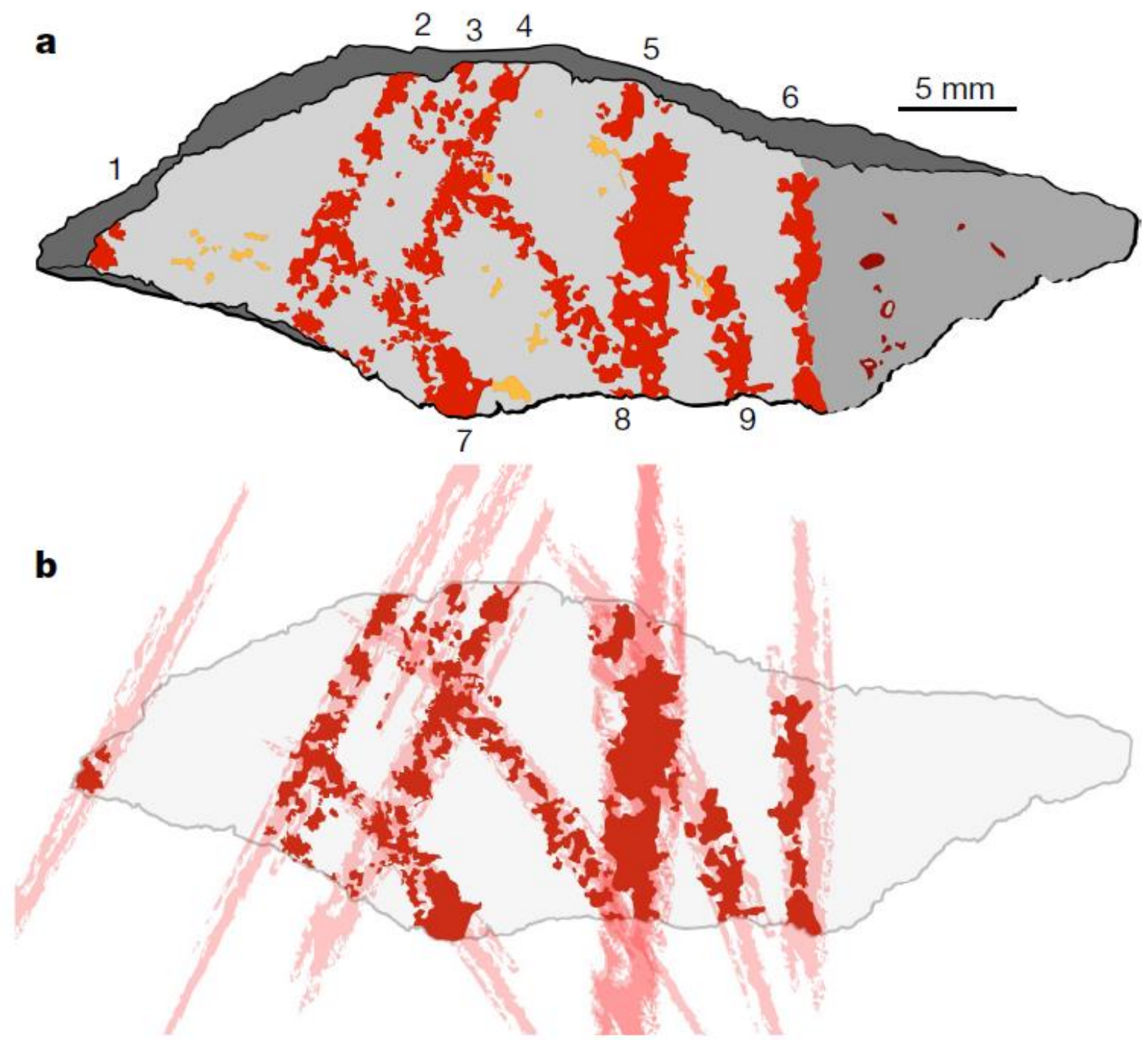

Fig. 3. Renditions of L13. a, Tracing with the drawn red lines numbered, the calcite patches shown in orange and the ochre residues in dark red. The ground surface is coloured light grey, the darker grey area indicates a flake scar and the darkest grey indicates the breakage fractures after L13 became detached from the originally larger grindstone. b, Schematic of lines extending beyond the outline of the present flake. 
Experimentally marking silcrete flakes with ochre crayons or paint indicates that the lines on L13 were produced with a crayon and thus constitute a drawing (Fig. 4, Extended Data Figs. 3, 4, Supplementary Information and Supplementary Table 1). Discontinuous lines consisting of loose powder in recesses and firmly adhering ochreous patches on elevated areas are produced when experimentally marking a silcrete flake with an ochre crayon. Parallel striations are visible on the patches. These same diagnostic features are seen on the lines composing the pattern on L13. By contrast, our experimental painted lines have clear edges, are solidly filled and show no striations (Extended Data Fig. 7a). The deposits produced by the two techniques are still clearly visible on the experimental material after rinsing under running water (Extended Data Fig. 7c). The width of the lines on the archaeological material, compared to their experimental counterpart, indicates that they were probably drawn with a pointed ochre crayon (Extended Data Fig. 5). Lines 2, 3, 4, 7, 8 and 9 were made by a single stroke with a 1.3-3.3-mm-wide ochre tip. Line 5 is wider and the ochre is more continuous, probably because it was created with multiple strokes. On experimental drawn lines, loose powder followed by a compacted streak is an indicator of stroke direction. When applied to the L13 drawing, this observation shows that the single-stroke lines 2, 3 and 4 were drawn in the same direction (Extended Data Figs. 3, 4). Line 5 is a multiple-stroke line, perhaps produced by a to-and-fro motion (Fig. 3). Line 6 does not show clear indication of directionality. Line 8-and probably lines 7 and 9-was drawn in a direction opposite to that of lines 2, 3 and 4, which could indicate that the object was turned during drawing. The order in which the lines were drawn could not be established. The surface on L13 with the drawing is smoothed by grinding and shows microscopic haematite-rich residues, which indicates that the object is a flake from a grindstone that was used to process ochre before the drawing was made (Extended Data Fig. 2). Subsequent cleaning of the grindstone by the inhabitants of BBC removed most traces of loose ochre powder (produced during grinding), and left a surface that was almost clean but which retained minute traces of ochre. 


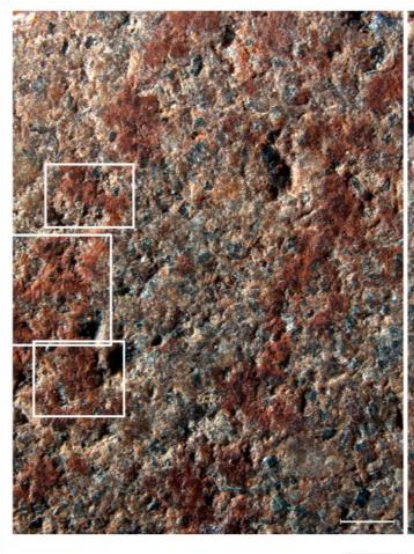

$\mathrm{mm}$

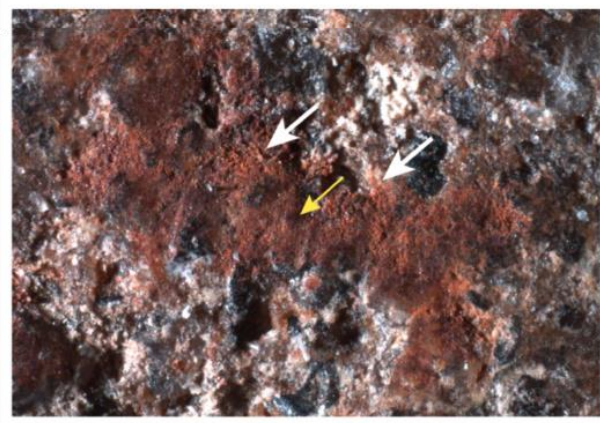

00

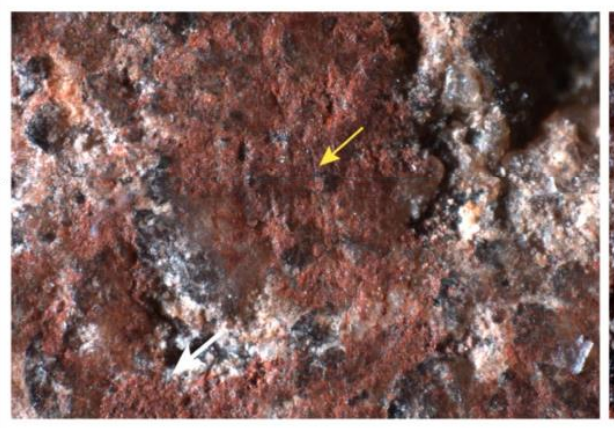

00

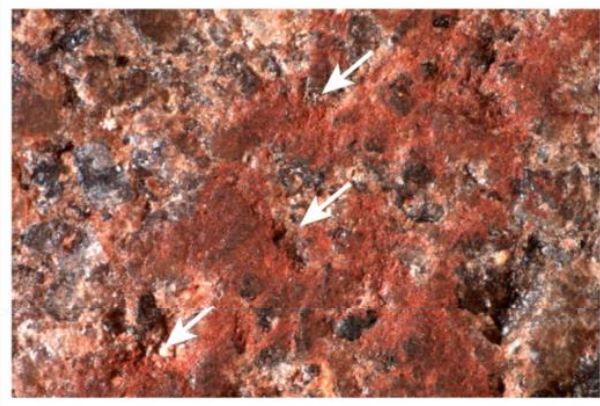

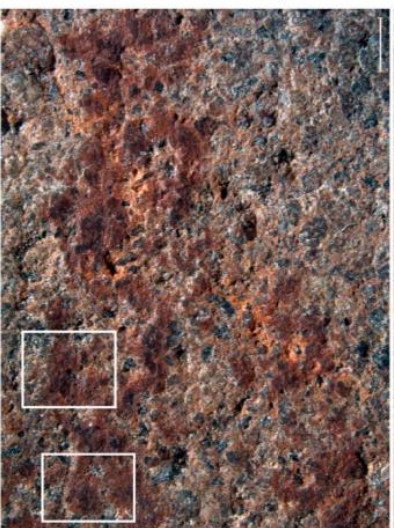

$\mathrm{mm}$

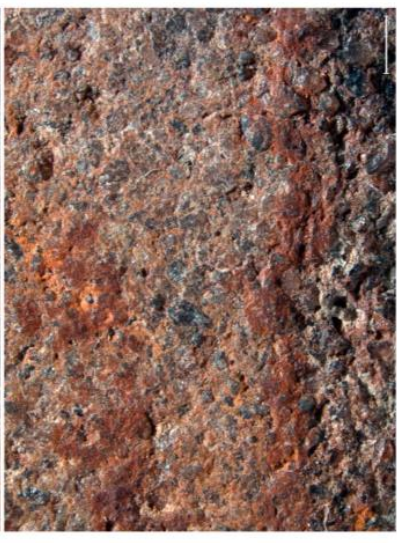

$\mathrm{mm}$

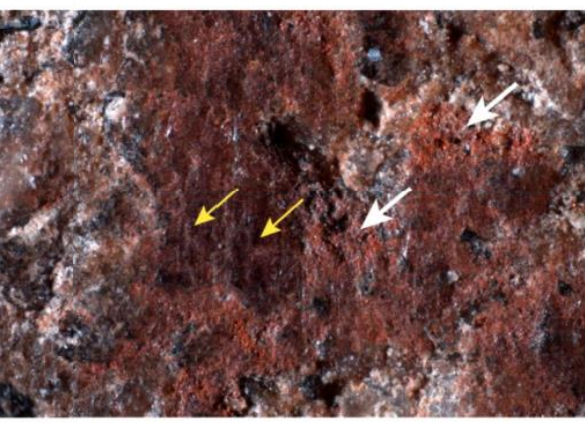

00

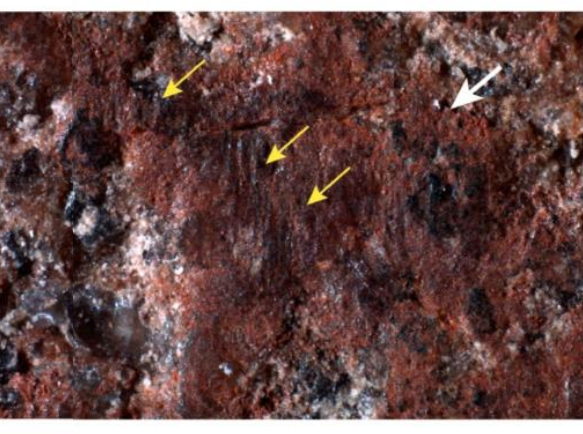

00
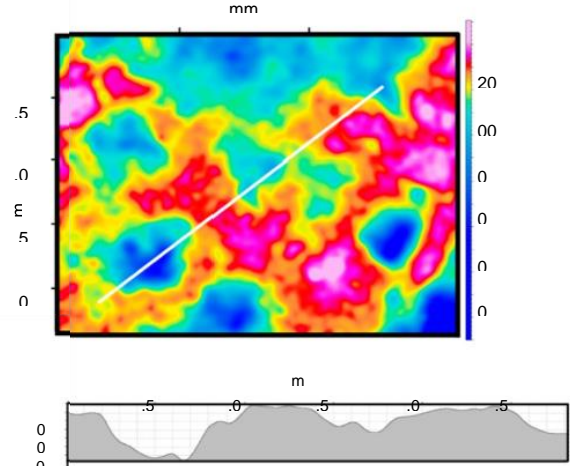

Fig. 4. Close-up views of the drawn lines on the L13 surface. a, Middle portions of lines 3 and 4 with location of the micrographs shown in $\mathrm{d}-\mathrm{f}$. b, Lines 8 and 9 on the proximal portion of the flake, with location of the micrographs g, h. c, Lines 9 and 6 on the proximal portion of the flake. i, Depth map of micrograph shown in f, with the location of the section shown in $\mathrm{j}$ indicated by a white line. Large white large arrows $(\mathrm{d}-\mathrm{h})$ point to deposits of powdery ochre preserved in recesses, small yellow arrows $(\mathrm{d}, \mathrm{e}, \mathrm{g}, \mathrm{h})$ point to prominent areas with compacted ochre deposits on which remnants of striations are still visible. These features are similar to those produced when a silcrete flake is experimentally marked with an ochre crayon (Extended Data Fig. 3b). 
Tribological analysis conducted with two different methods supports the grindstone hypothesis; it shows that the surface with the drawing is significantly smoother than the other faces of L13 and typical cortical and knapped surfaces of other pieces of silcrete recovered from MSA levels at BBC, which confirms that the smoothing recorded on L13 cannot be due to natural processes (Extended Data Fig. 6a, b, Supplementary Information and Supplementary Table 3). Experimental reproduction of the lines found on L13 using the same technique (that is, using a pointed ochre crayon) indicates that the drawing was more visible when it was produced and that the loose powder that originally composed the lines was subsequently lost through taphonomic processes and rinsing. The lines produced on smooth silcretesuch as on L13-are better defined than on rough surfaces; and the ochre crayon used for the design was probably soft and produced lines that adhered well to the silcrete. We conclude that the ochre crayon was intentionally used to produce a cross-hatched design. Ochre powder was used for practical purposes by MSA people; for example, as a glue additive and perhaps as a sun screen12,13. Experimental reproduction of numerous lines longer than all those on L13 (Extended Data Fig. 3b, Supplementary Information) produced less than $1 \mathrm{mg}$ of ochre powder, thus discounting a utilitarian objective for the production of the L13 lines.

Abstract engravings are known from several archaeological sites that pre-date the Later Stone Age (which began about 42-44 ka) in Africa, and from the Upper Palaeolithic (dated to about $44 \mathrm{ka}$ ) in Europe; these include an engraved shell from Trinil dated to $540 \mathrm{kal4}$, an engraved bone from Bilzingsleben at $370 \mathrm{ka15}$, engraved ochre from BBC (100-73 ka)11, engraved cortices from Qafzeh (90 ka)16 and Quneitra (60 ka)17, engraved ostrich egg shells from Diepkloof18 and Klipdrift Shelter (65$59 \mathrm{ka}) 19$ and engraved bedrock from Gorham's Cave (over $40 \mathrm{ka}$ )20. A date of 66-64 ka-which would indicate Neanderthal authorship-has recently been proposed for ochre markings from three caves in Spain21,22. However, the earliest previously known evidence for drawing techniques comes from much younger sites that post-date $42 \mathrm{ka}$, such as Chauvet23,24, El Castillo25, Apollo 1126 and Maros caves27. The discovery of L13 demonstrates that drawing was part of the behavioural repertoire of populations of early Homo sapiens in southern Africa at about $73 \mathrm{ka}$. It demonstrates their ability to apply similar graphic designs on various media using different techniques. The discovery of abstract engravings on ochre, with patterns comparable to L13, from levels at BBC dated to 100-73 ka (Fig. 1) and the production of an ochre-rich paint stored in abalone shells6 suggest that drawings and possibly paintings may have been produced in older MSA levels, perhaps since $100 \mathrm{ka}$. The cross-hatched pattern of L13 pre-dates by at least 30,000 years the earliest previously known abstract and figurative drawings. This finding supplements previous evidence reflecting cultural modernity and symbol use that has already been identified in the MSA levels at BBC through the discovery of personal ornaments, elaborate bone tools, engravings, and the production and storage of pigmented compounds. The L13 drawing adds a further dimension to our understanding of the processes that shaped the behaviour and cognition of early H. sapiens.

\section{Methods}

L13 was photographed using various macro-lenses, and examined and photographed with a motorized Leica Z6 APOA equipped with a DFC420 digital camera linked to LAS Montage and Leica 
Map DCM 3D computer software. Sections and three-dimensional models of selected portions of the lines on L13 were obtained with the LAS Montage, or by exporting depth maps obtained with the LAS Montage into the Leica Map DCM 3D. An image of the surface with the cross-hatching was obtained by importing overlapping micrographs from the LAS Montage into Adobe Illustrator and then producing a tracing of the red patches and other features identified on L13. This tracing was compared to the original under a microscope and corrected as required. Data on the morphology, size, number of patches composing the lines on L13 and presence of striations on these lines were recorded.

Unmodified pieces of ochre (Extended Data Fig. 7a) with narrow pointed or linear edges and variable texture and hardness were used to experimentally mark silcrete flakes (Extended Data Fig. 7b). These flakes are archaeological objects that derive from weathered and eroding MSA levels on the BBC talus.

The silcrete flakes were carefully cleaned with a brush under running water between marking experiments to remove all traces of ochre from their surfaces. In the first experiment, both pointed and linear ochre pieces were used to produce single- and multiple-stroke straight and curved lines. Multiple strokes were produced by repeatedly passing the ochre edge over the silcrete surface in the same direction or with a to-and-fro movement in an effort to accurately superimpose each new line on those previously made. In a second experiment, four pointed and four linear ochre edges were used to produce a sequence of six single-stroke, parallel, straight four-centimetre-long lines per edge (Supplementary Table 1).

The width and length of the microfacets created on the ochre pieces by the marking process and the maximum and minimum width of the lines produced on the silcrete flakes were measured with a digital calliper after each line was produced. In a third experiment, fine-grained ochre powder, produced by rubbing ochre pieces on a grindstone, was mixed with water in three different concentrations to produce three gradations of viscosity (thin, medium and thick).

Wooden sticks of $1 \mathrm{~mm}$ and $2 \mathrm{~mm}$ in diameter were gently crushed at one end with a hammer over a length of $1 \mathrm{~cm}$ to create a brush, and this was used to apply the three types of paint onto flat surfaces on the silcrete flakes.

The experimental lines made with the ochre pieces and wooden applicators were examined and photographed with the same equipment and procedures used for examining L13 immediately after their production and after being gently washed for $10 \mathrm{~s}$ under running tap water and dried at air temperature. This is the same cleaning process used for L13 and other BBC lithics recovered during sieving. When examining the experimental drawn and painted lines under the microscope, particular attention was paid to identifying features diagnostic of the marking technique, the direction in which the lines were made, the type of ochre edge used and the way in which washing altered the lines. In a final experiment, loose ochre powder produced by drawing nine four-centimetre-long straight lines with pointed and linear pieces on a silcrete flake was recovered and weighed on a scale with an accuracy of $1 \mathrm{mg}$.

Scanning electron microscopy with energy-dispersive X-ray spectroscopy (SEM-EDS) analyses were performed using a FEI Quanta 200. Back-scattered electron images (BSE) and elemental analyses were conducted under a low vacuum mode with an accelerating voltage of $15 \mathrm{kV}$. BSE images were produced with a SiLi detector and EDS analyses with a SDD-EDAX detector. The EDS analyses were conducted under similar magnifications $(\times 50, \times 100$ or $\times 200$, and $\times 500$ or $\times 1,000)$, at the same working 
distance $(10 \mathrm{~mm})$ and with the same acquisition time (100 s) for each EDS spectrum. Semi-quantitative data were calculated in weight percentages and normalized to $100 \%, \mathrm{C}$ and $\mathrm{O}$ being included. Major $(>10 \%)$, minor $(>3 \%)$ and minor-to-trace elements $(<3 \%)$ were distinguished ${ }^{28}$. For Raman analysis we used a SENTERRA dispersive Raman microscope (Bruker), equipped with an internal calibration system. The analyses were done with a 785-nm laser and a power of $1 \mathrm{~mW}$ to avoid transformation of mineral phases. Acquisition time was set to between $30 \mathrm{~s}$ and $70 \mathrm{~s}$, and several co-additions of the signal if necessary. The working area was observed through the integrated colour camera, and data were collected with the software package OPUS 7.2.

High-resolution surface topography was acquired with a Sensofar S neox confocal microscope driven by SensoScan 6 software (Sensofar). For this study, we selected thirty $1.67 \times 1.25-\mathrm{mm}$ areas that were measured with a $20 \times$ objective (NA 0.45). This allowed for a spatial resolution of $0.65 \mu \mathrm{m}$ and a vertical resolution of $0.31 \mu \mathrm{m}$. Seven areas were measured outside of the red lines on the surface with the drawing, seven on the other surfaces of L13, ten on the knapped surfaces and ten on the natural cortical surfaces of two fine-grained silcrete flakes from the MSA levels at BBC (Extended Data Fig. 6a). Three-dimensional reconstructions of the measured areas were visually compared. The location of the measured areas on L13 was randomly selected (Extended Data Fig. 6c). Tribological analysis of these surfaces entailed pre-treatments and the calculation of surface texture parameters according to the ISO standard 25178, using the SensoMap software. Pre-treatments conducted on the raw acquisitions consisted of (1) filling non-measured points using the neighbourhood valid points algorithm, (2) removing the general shape of the surface by subtracting a second-degree polynomial, (3) separating waviness from roughness by the application of a Gaussian filter with a $0.25-\mathrm{mm}$ cut-off value, so that only roughness is kept for the calculation of surface texture parameters. Height, functional, spatial, hybrid and functional volume parameters were calculated for each quarter of each pre-treated area. Kruskall-Wallis multiple comparison test ${ }^{29}$ were applied to the dataset.

Scale sensitive fractal analysis using SensoMap 7.4.8443 software was also applied to L13 ${ }^{30}$. This analysis calculates the difference between the topographic surface and its planimetric area. The calculation is made at many consecutive scales by reducing the area of the triangles tiling the surface. Results are represented by an s-curve in which the rising indicates the scale at which the surface becomes rough.

A high-resolution three-dimensional model was created by photogrammetry using the Photoscan Standard software (Agisoft, version 1.4.0). Fifty-six photos taken with a macro lens from different points of view were processed to create the model and apply the texture (Supplementary Data and Supplementary Video).

\section{Acknowledgements}

Partial funding for this research was provided to C.S.H., K.L.v.N. and F.d'E. by the Research Council of Norway through its Centres of Excellence funding scheme, Centre for Early Sapiens Behaviour (SapienCE), project number 262618; to C.S.H. by a South African National Research Foundation Research Chair (SARChI) at the University of the Witwatersrand and the Evolutionary Studies Institute at the University of the Witwatersrand, and the University of Bergen, Norway; F.d'E., L.D. and A.Q. by the LaScArBx, a research programme supported by the ANR (ANR-10-LABX-52). We thank C. Foster for the image in Fig. 2; P. Keene for assistance in the Cape Town laboratory, I. Svahn for assistance with electron microscopy in Bordeaux, G. Devilder for his input on Fig. 3 and M. Haaland for his stratigraphy image on Fig. 1. 


\section{References}

1. d'Errico, F. \& Stringer, C. B. Evolution, revolution or saltation scenario for the emergence of modern cultures? Phil. Trans. R. Soc. Lond. B 366, 1060-1069 (2011).

2. Henshilwood, C. S. et al. Emergence of modern human behavior: Middle Stone Age engravings from South Africa. Science 295, 1278-1280 (2002).

3. Henshilwood, C., d'Errico, F., Vanhaeren, M., van Niekerk, K. \& Jacobs, Z. Middle Stone Age shell beads from South Africa. Science 304, 404 (2004).

4. d'Errico, F. \& Henshilwood, C. S. Additional evidence for bone technology in the southern African Middle Stone Age. J. Hum. Evol. 52, 142-163 (2007).

5. Mourre, V., Villa, P. \& Henshilwood, C. S. Early use of pressure flaking on lithic artifacts at Blombos Cave, South Africa. Science 330, 659-662 (2010).

6. Henshilwood, C. S. et al. A 100,000-year-old ochre-processing workshop at Blombos Cave, South Africa. Science 334, 219-222 (2011).

7. Jacobs, Z., Hayes, E. H., Roberts, R. G., Galbraith, R. F. \& Henshilwood, C. S. An improved OSL chronology for the Still Bay layers at Blombos Cave, South Africa: further tests of single-grain dating procedures and a re-evaluation of the timing of the Still Bay industry across southern Africa. J. Archaeol. Sci. 40, 579-594 (2013).

8. Villa, P., Soressi, M., Henshilwood, C. S. \& Mourre, V. The Still Bay points of Blombos Cave (South Africa). J. Archaeol. Sci. 36, 441-460 (2009).

9. d'Errico, F., Henshilwood, C. S. \& Nilssen, P. An engraved bone fragment from c. 70,000-year-old Middle Stone Age levels at Blombos Cave, South Africa: implications for the origin of symbolism and language. Antiquity 75, 309-318 (2001).

10.Vanhaeren, M., d'Errico, F., van Niekerk, K. L., Henshilwood, C. S. \& Erasmus, R.

M. Thinking strings: additional evidence for personal ornament use in the Middle Stone Age at Blombos Cave, South Africa. J. Hum. Evol. 64, 500-517 (2013).

11.Henshilwood, C. S., d'Errico, F. \& Watts, I. Engraved ochres from the Middle Stone Age levels at Blombos Cave, South Africa. J. Hum. Evol. 57, 27-47 (2009).

12.Wadley, L. Ochre crayons or waste products? Replications compared with MSA 'crayons' from Sibudu cave, South Africa. Before Farming 2005, 1-12 (2005).

13.Rifkin, R. F. et al. Evaluating the photoprotective effects of ochre on human skin by in vivo SPF assessment: implications for human evolution, adaptation and dispersal. PLOS ONE 10, e0136090 (2015).

14.Joordens, J. C. et al. Homo erectus at Trinil on Java used shells for tool production and engraving. Nature 518, 228-231 (2015).

15.Mania, D. \& Mania, U. Deliberate engravings on bone artefacts of Homo erectus. Rock Art Res. 5, 91-107 (1988).

16.Hovers, E., Vandermeersch, B. \& Bar-Yosef, O. A Middle Palaeolithic engraved artefact from Qafzeh Cave, Israel. Rock Art Res. 14, 79-87 (1997).

17.Marshack, A. A Middle Paleolithic symbolic composition from the Golan heights: the earliest known depictive image. Curr. Anthropol. 37, 357-365 (1996).

18.Texier, P.-J. et al. The context, form and significance of the MSA engraved ostrich eggshell collection from Diepkloof Rock Shelter, Western Cape, South Africa. J. Archaeol. Sci. 40, 34123431 (2013).

19.Henshilwood, C. S. et al. Klipdrift Shelter, southern Cape, South Africa: preliminary report on the Howiesons Poort layers. J. Archaeol. Sci. 45, 284-303 (2014).

20.Rodríguez-Vidal, J. et al. A rock engraving made by Neanderthals in Gibraltar. Proc. Natl Acad. Sci. USA 111, 13301-13306 (2014).

21.Hoffmann, D. L. et al. U-Th dating of carbonate crusts reveals Neandertal origin of Iberian cave art. Science 359, 912-915 (2018).

22.Pearce, D. G. \& Bonneau, A. Trouble on the dating scene. Nat. Ecol. Evol. 2, 925-926 (2018).

23.Quiles, A. et al. A high-precision chronological model for the decorated Upper Paleolithic cave of Chauvet-Pont d'Arc, Ardèche, France. Proc. Natl Acad. Sci. USA 113, 4670-4675 (2016).

24.Sadier, B. et al. Further constraints on the Chauvet cave artwork elaboration. Proc. Natl Acad. Sci. USA 109, 8002-8006 (2012).

25.Pike, A. W. et al. U-series dating of Paleolithic art in 11 caves in Spain. Science 336, 1409-1413 (2012).

26.Wendt, W. E. 'Art mobilier' from the Apollo 11 Cave, South West Africa: Africa's oldest dated works of art. S. Afr. Archaeol. Bull. 31, 5-11 (1976). 
27.Aubert, M. et al. Pleistocene cave art from Sulawesi, Indonesia. Nature 514, 223-227 (2014).

28.d'Errico, F. et al. The technology of the earliest European cave paintings: El Castillo Cave, Spain. J. Archaeol. Sci. 70, 48-65 (2016).

29.Siegel, S. \& Castellan, N. J. Jr. Nonparametric Statistics for the Behavioral Sciences (McGrawHill, New York, 1988).

30.Brown, C. A. in Characterisation of Areal Surface Texture (ed. Leach, R.) 129-153 (Springer, Berlin, 2013). 
A

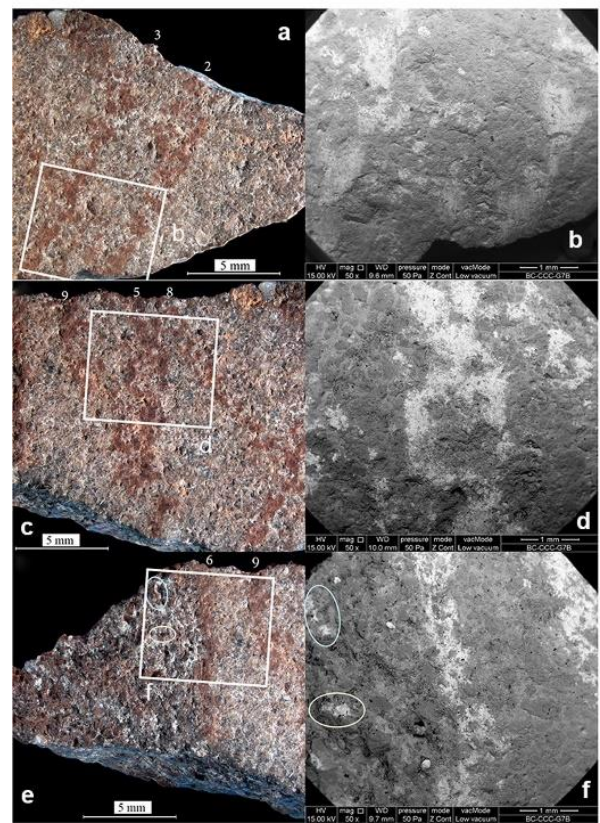

B

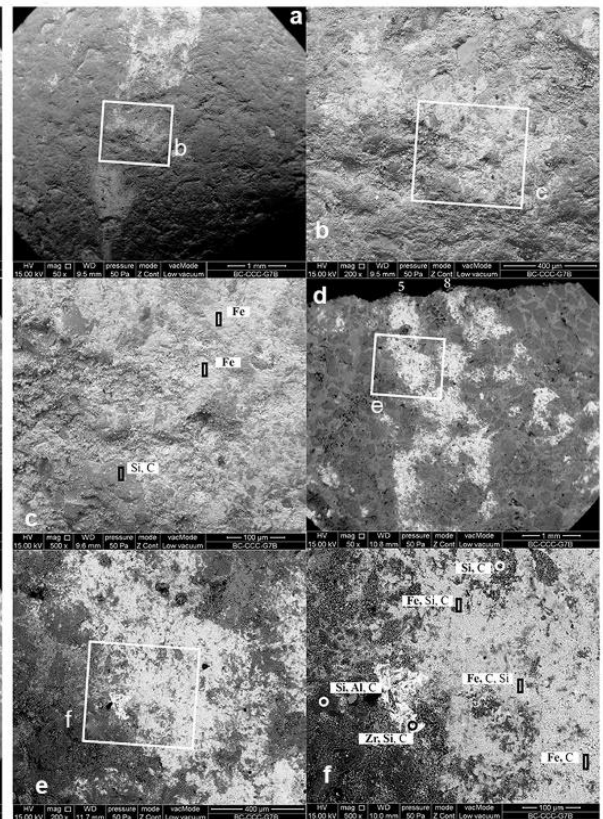

C
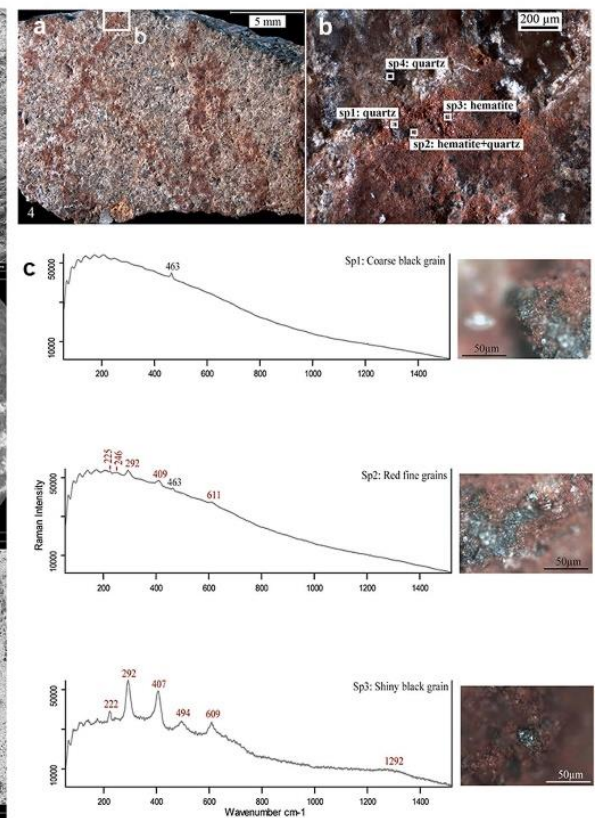

Extended Data Fig. 1. Microscopic examination and chemical analyses of the juxtaposed patches of red deposit that form the drawn lines on L13 and the smoothed surface of the silcrete flake. The lines consist mainly of fine-grained iron oxide (Fe), that were applied to the surface, as no haematite occur naturally in the silcrete raw material of L13. A, Photographs (left) and SEM-EDS images (right) of the red lines of the surface of L13. In the subpanels of A, images in a, b show lines 2 and 3; c, $\mathbf{d}$ show lines 5, 8 and 9; and e, $\mathbf{f}$ show lines 6 and 9 and red spots on a flake scar. The white rectangles in a, $\mathbf{c}$ and $\mathbf{e}$ indicate the areas that are enlarged in subpanels $\mathbf{b}, \mathbf{d}$ and $\mathbf{f}$, respectively. Notice the white appearance of the lines in the backscattered electrons SEM-EDS images due to the presence of iron rich deposits. B, SEM-EDS images (back-scattered electrons) of line 2 and 5. Subpanels a-c show line 2; $\mathbf{d}-\mathbf{f}$ show line 5. White squares indicate areas that are enlarged in the image with the corresponding letter. The rectangles and black/white circles in subpanels $\mathbf{c}, \mathbf{f}$ show differences in elemental composition between the drawn lines (light areas) and the silcrete surface (dark areas). C, Raman analysis of line 4. Subpanel a shows a photograph with the location of the analysed area (white rectangle). Subpanel b shows the analysed spots and identified minerals. Subpanel $\mathbf{c}$ shows Raman spectra and micrographs of the analysed areas with peaks identifying haematite (red numbers) and quartz (black numbers). 
A

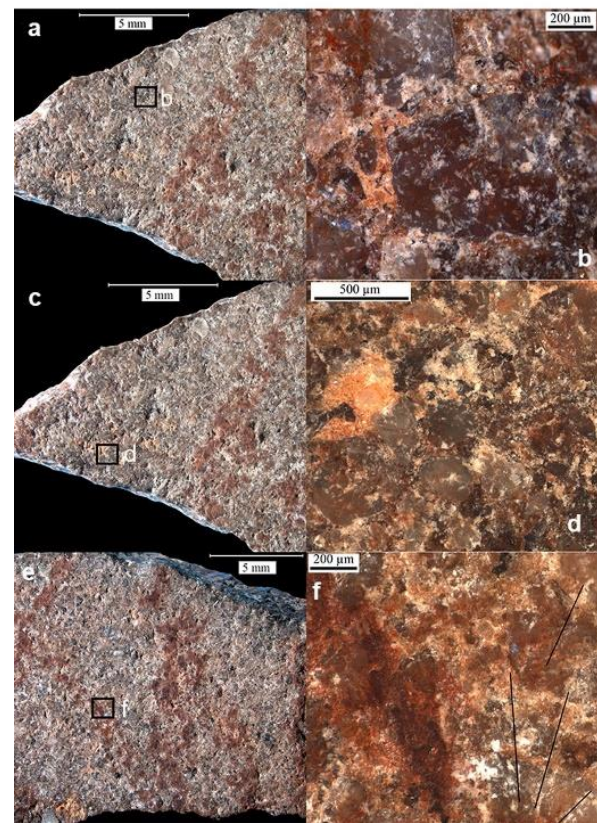

B

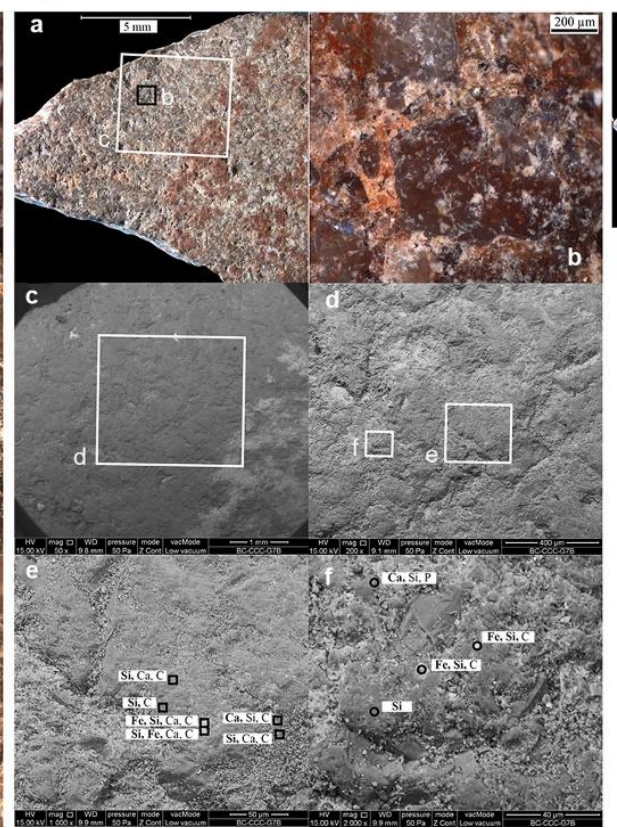

C
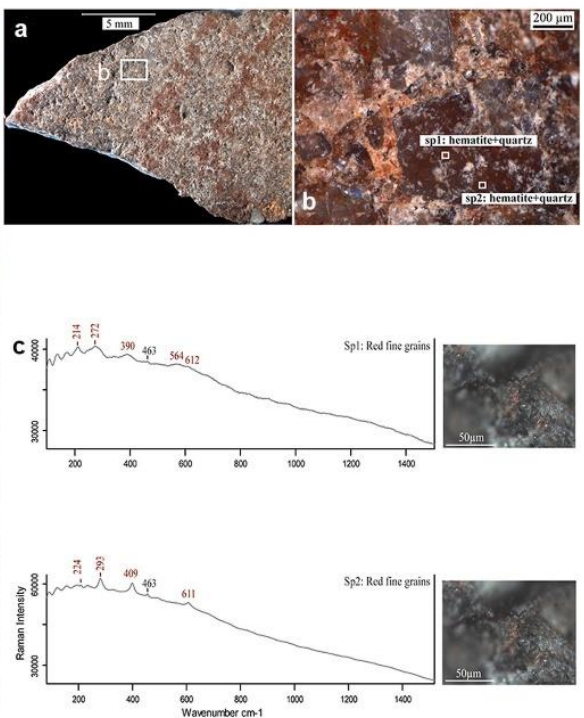

Extended Data Fig. 2. Microscopic examination and chemical analyses of microresidues. The microresidues on the smoothed silcrete surface outside of the lines differ in Fe content from the red lines, which-along with the presence of microstriations - supports the theory that the silcrete flake was part of an ochre grindstone before the drawing was made. A, Photographs and micrographs of the lines drawn on L13. Black squares in subpanels a, $\mathbf{c}$, e indicate the areas enlarged in the adjacent subpanels $\mathbf{b}, \mathbf{d}, \mathbf{f}$. Red residues are clearly visible on the matrix and on quartz grains. f, Black lines highlight superficial randomly oriented striations. B, SEM-EDS analysis of the silcrete outside the drawn lines. In subpanels a, c, d, black and white squares indicate the areas enlarged in the adjacent photograph. In subpanels $\mathbf{e}, \mathbf{f}$, the analysed spots (black squares and circles) identify the presence of isolated iron-rich particles on the surface of the matrix and the quartz grains. C, Raman analysis of microresidues preserved in quartz grain pits. Subpanel a shows a photograph with the location of the analysed area (white rectangle). Subpanel b shows analysed spots (white squares) and identified minerals. Subpanel c shows Raman spectra and micrographs of the analysed areas, with peaks identifying haematite (red numbers) and quartz (black numbers). The area shown in subpanel $\mathbf{b}$ of panel $\mathbf{C}$ is the same as the area shown in subpanel $\mathbf{b}$ of panel $\mathbf{B}$. 
A

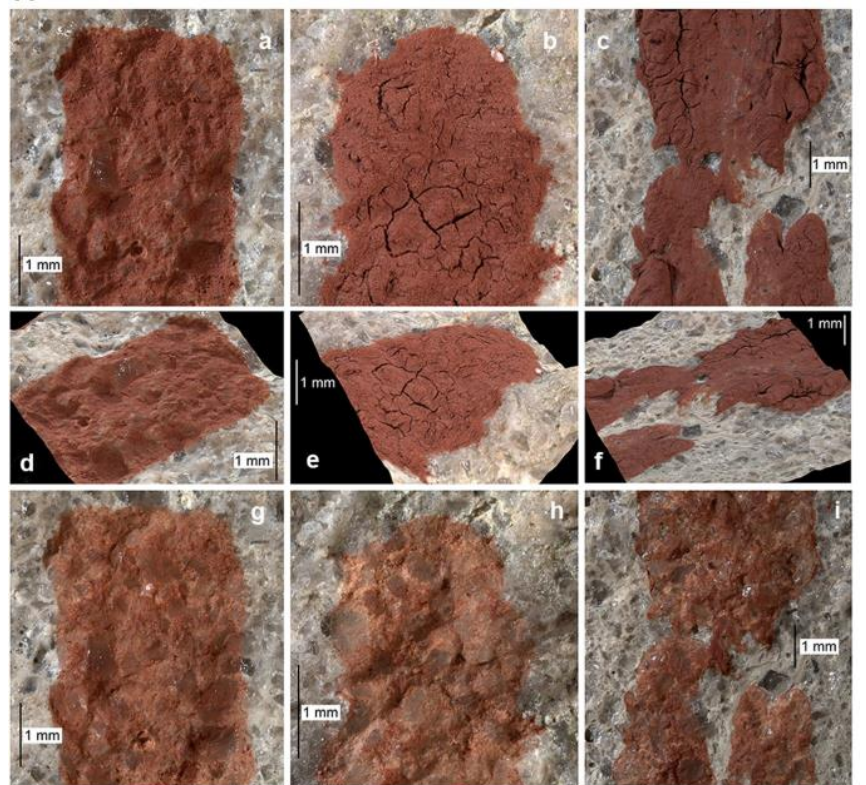

B
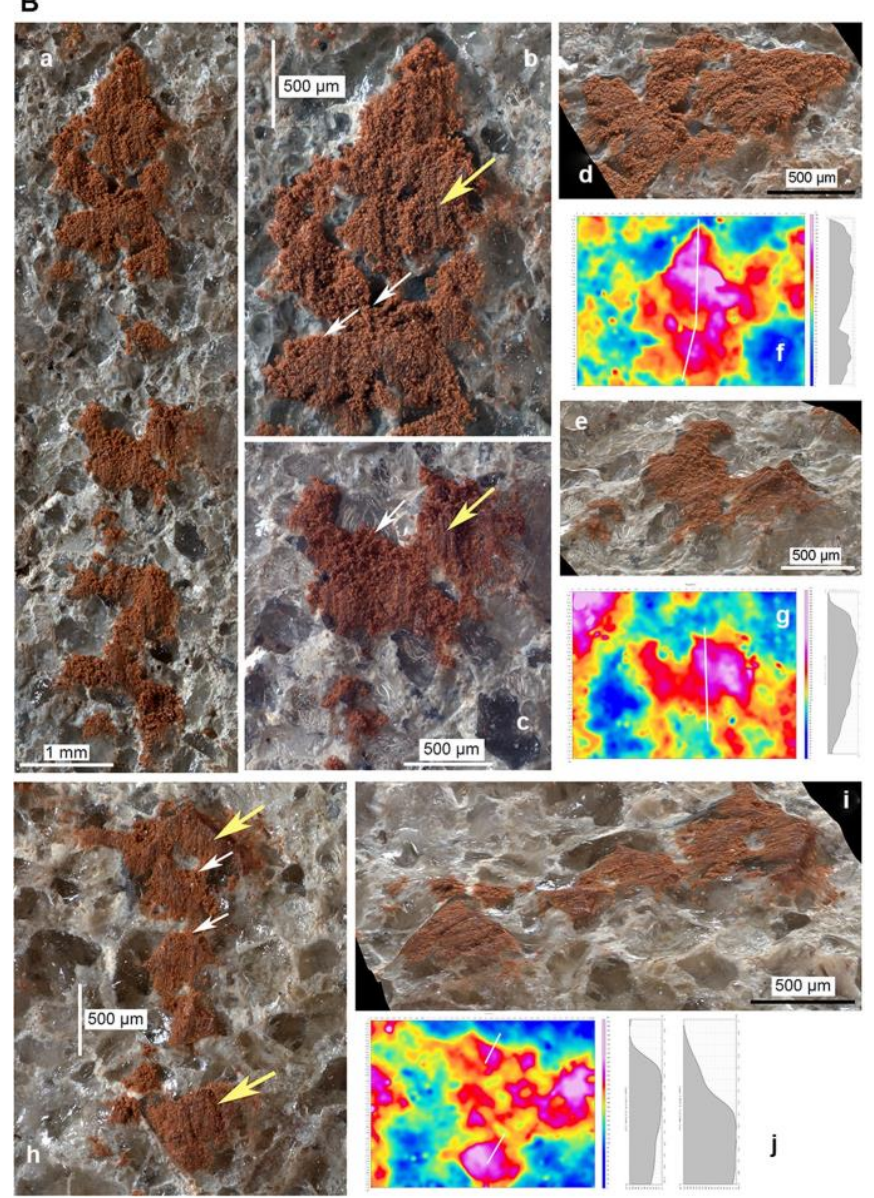

Extended Data Fig. 3. Results from experimental marking of silcrete surfaces with ochre paint of varying viscosities and with an ochre crayon, and subsequent rinsing. A, Micrographs of experimentally painted lines before and after rinsing. Subpanels a-c show lines produced by applying a liquid (a), viscous (b) and very viscous (c) paint with a thin wooden brush on a silcrete surface. Subpanels $\mathbf{d}-\mathbf{f}$ show the threedimensional rendering of the same lines showing the surface topography. Subpanels $\mathbf{g}-\mathbf{i}$ show the same lines after gently rinsing the surface of the silcrete under running tap water. $\mathbf{B}$, Lines produced experimentally on a silcrete flake with an ochre crayon. Subpanel a shows a single-stroke line drawn from the top to the bottom. Subpanels b, c show close-up views and three-dimensional renderings (subpanels d, e) of selected areas of subpanel a. Subpanels $\mathbf{h}$, i show a photograph (h) and three-dimensional rendering (i) of a single-stroke line produced from the top to the bottom after gently rinsing the silcrete flake under running tap water. Subpanels $\mathbf{f}, \mathbf{g}$ and $\mathbf{j}$ are depth maps and sections of $\mathbf{b}, \mathbf{c}$ and a selected area of $\mathbf{h}$, respectively. The locations of the sections are indicated on the depth maps by white bars. White arrows indicate deposits of powdery ochre preserved in recesses, and larger yellow arrows indicate prominent areas with compacted ochre deposits covered by striations. Compacted patches of ochre covered by striations and small deposits of ochre powder in recesses are preserved after the rinsing; these features and lines are similar to those on L13 (Fig. 4). 


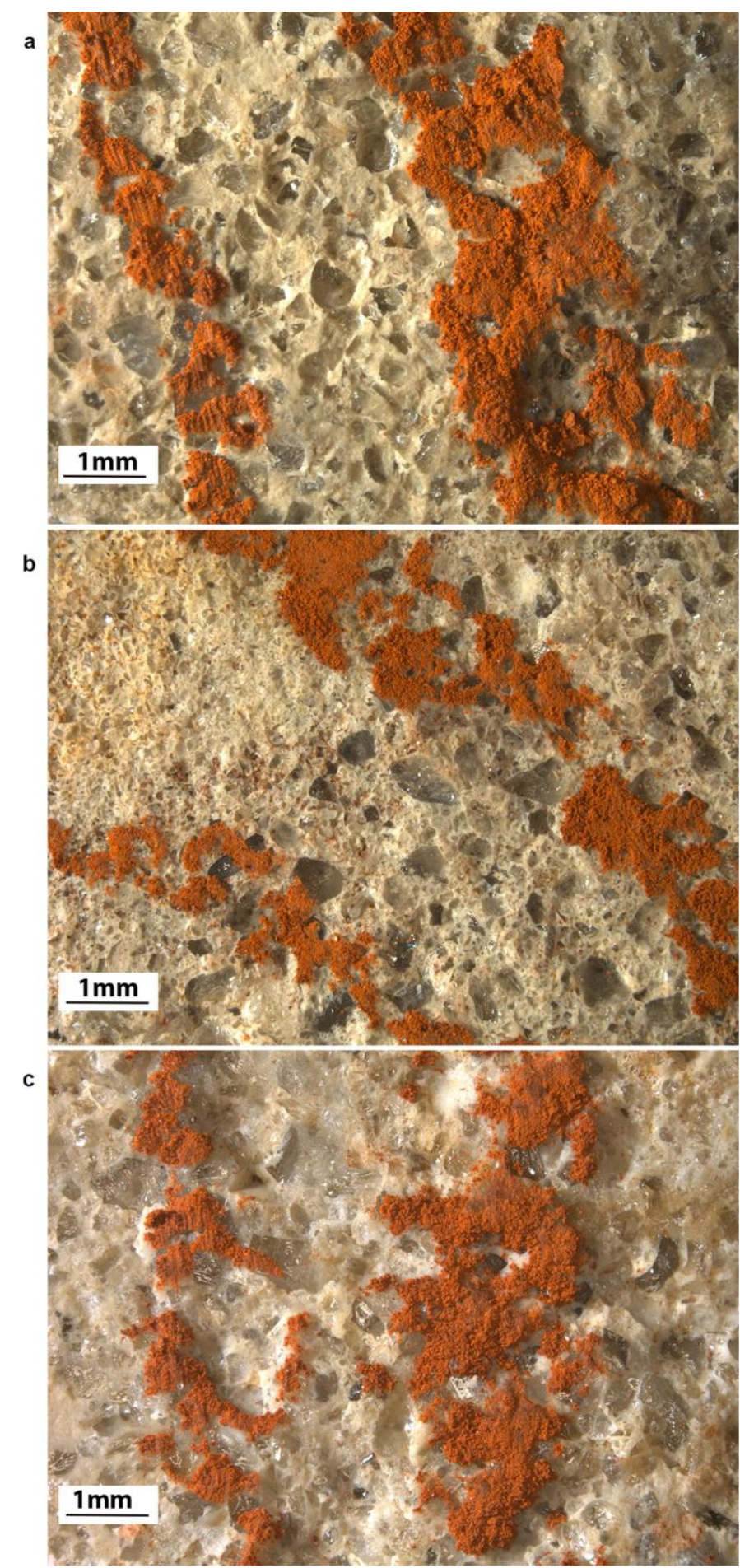

Extended Data Fig. 4. Lines produced experimentally on silcrete flakes. These images show that, as it is difficult to exactly superimpose a new line on a previous one, superimposing a line on a previous line generally results in a wider line. Unidirectional, superimposed lines retain the same features observed on a single-stroke line. Multiple lines produced by a toand-fro movement of the ochre edge show microscopic evidence that the crayon was moved in both directions. a, Straight single- (left) and five-stroke line (right) produced from the top to the bottom. b. Curved single- (left) and five-stroke line (right) produced from top left to the bottom right. c, Straight single- (left) and five-stroke line (right) produced by a to-and-fro motion. The lines in this figure were not rinsed with water. 

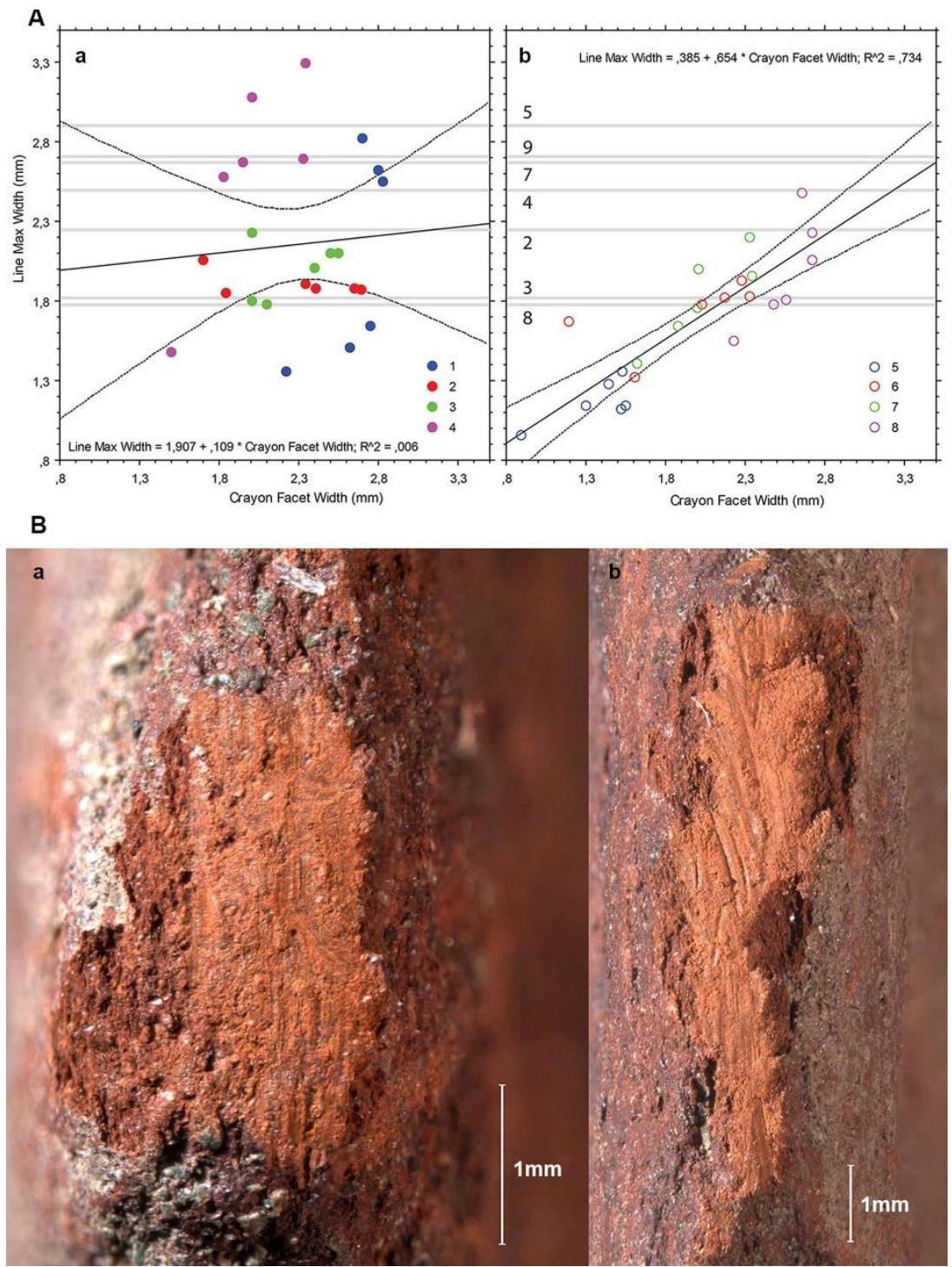

Extended Data Fig. 5. Experimental marking of silcrete flakes with a variety of ochre crayons. The morphology of lines will depend on the properties and composition of the ochre, the roughness of the silcrete surface, the pressure exerted and the morphology of the ochre area in contact with the silcrete. In general, soft, plastic, clayish ochre will produce thicker and more continuous lines than silty or sand-rich ochre. Lines on fine-grained silcrete will be better defined than those on coarse silcrete. Stronger pressure will produce comparatively wider, thicker and better defined lines. Six lines made with each of eight unmodified ochre crayons had a maximum width ranging from about 0.9 to $3.3 \mathrm{~mm}$. Lines produced with a pointed ochre crayon tend to be wider and more variable in width than those made with a linear edge. The width of lines made with a linear edge is strongly correlated with the maximum width of the facets on the ochre piece. By contrast, no correlation is observed between the lines made with pointed crayons and the maximum width of the facets on the crayon. The width of the lines on the drawn cross-hatching present on L13 is comparable with that of the experimental lines. The range (1.8-2.9 mm) of this width best fits the width variability observed when marking the silcrete with a pointed crayon rather than an edge. This indicates that a pointed ochre crayon was used to produce the cross-hatching and that the facet of the crayon in contact with the silcrete was about 1.3-2.9 mm wide. A, Correlation between the width of lines and the width of the resulting facets on eight experimental ochre crayons. Subpanel a shows results from crayons with pointed active areas. Subpanel b shows results from crayons with linear active areas. The grey bars indicate the width of lines on L13. B, Wear facet appearing on the natura surface of an ochre crayon after a single stroke (subpanel a) and five strokes (subpanel b). 
A
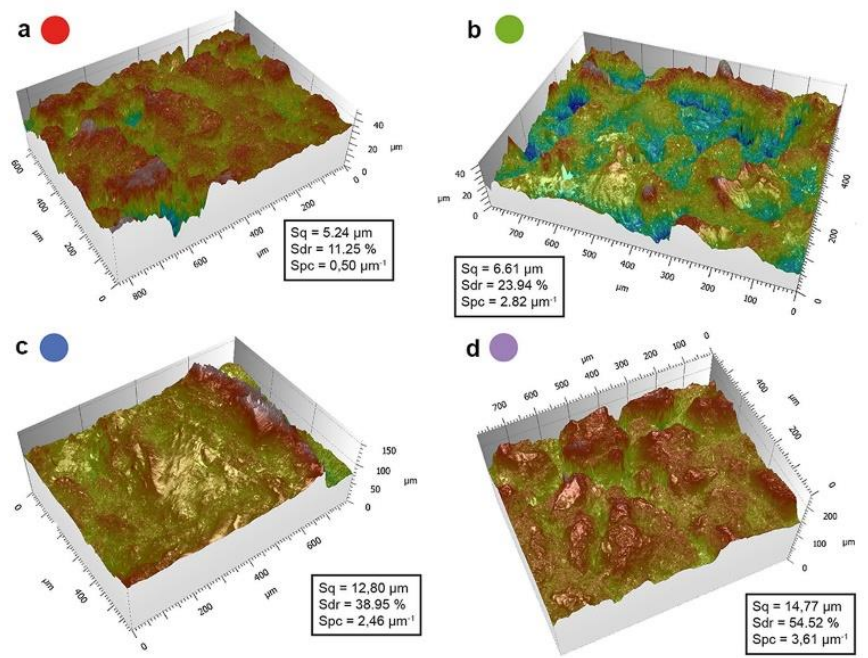
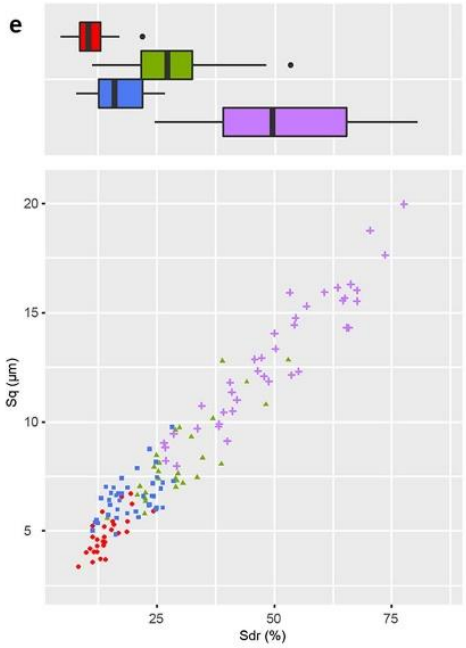

$B B C L 13$ - drawn surface $(n=28)$

BBC L13 - other surface $(n=28)$

- BBC MSA flakes - knapped surfaces $(n=40)$

$+\quad B B C$ MSA flakes - cortical surfaces $(n=40)$

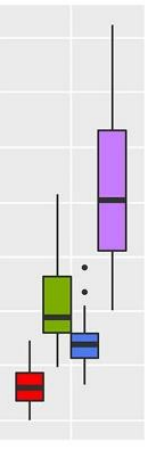

B

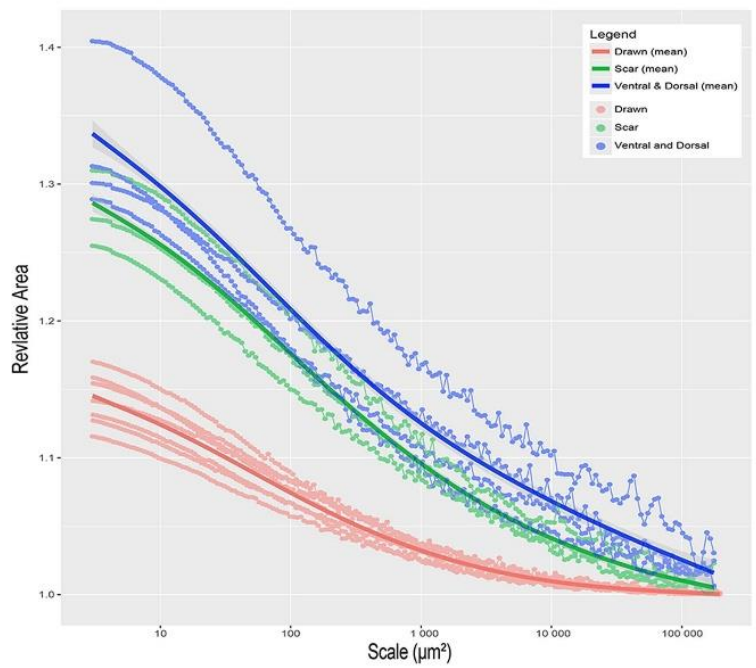

C

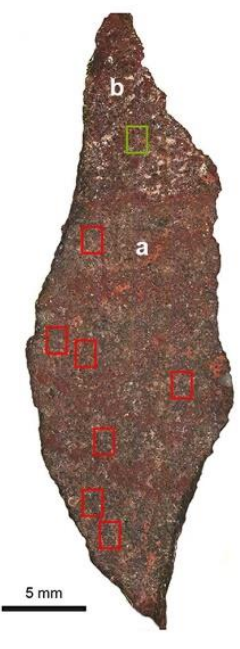

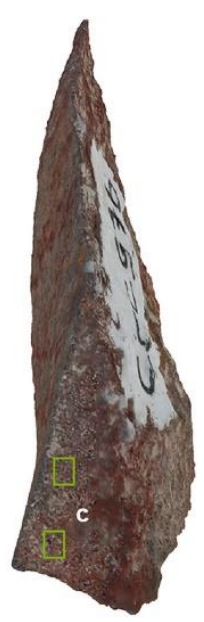

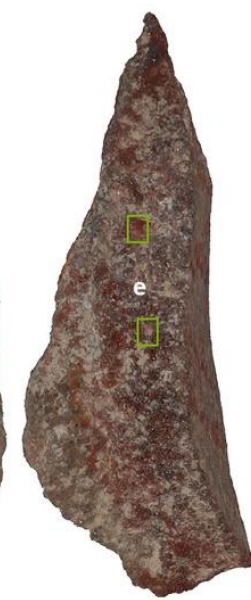

Extended Data Fig. 6. Three-dimensional rendering of microscopic areas of L13 and silcrete flakes from BBC. Threedimensional rendering shows flattening of the surface of L13 with the drawing, dissolution of the matrix between quartz grains on the cortex of the BBC silcrete flakes and an unworn appearance of the other surfaces of L13 and the ventral aspect of the BBC silcrete flakes. A, Roughness analysis of L13 and MSA silcrete flakes from BBC. Subpanels a-d show three-dimensional renderings of a selected area of the surface of L13 with the drawing (a), other surfaces of L13 (b), knapped (c) and cortical (d) surfaces of BBC silcrete flakes. Subpanel e shows box plots of the variation of roughness variables Sq and Sdr, and a bi-plot correlating these two variables. Notice the high degree of smoothness of the surface of L13 with the drawing relative to the other surfaces. A Kruskall-Wallis multiple comparison test demonstrates that Sq, Sdr and Spc on the surface with the drawing are significantly lower $(P<0.01)$ than those measured on the remainder of the analysed surfaces of L13. B, Areal fractal analysis confirms a clear difference in roughness between the surface with the drawing and other surfaces of L13. This is consistent with the interpretation of the wear on the surface with the drawing as being produced by grinding activities before the drawing occurred. C, Analysis of L13 with confocal microscopy. Rectangles indicate the locations of the analysed areas on the surface with the drawing (red) and on the other surfaces (green). Letters distinguish analyses conducted with areal fractal analysis on the surface with the drawing (a), a flake scar on the surface with the drawing (b), a flake scar on the dorsal surface (c) from analyses made on the dorsal (d) and ventral surfaces (e). 
a
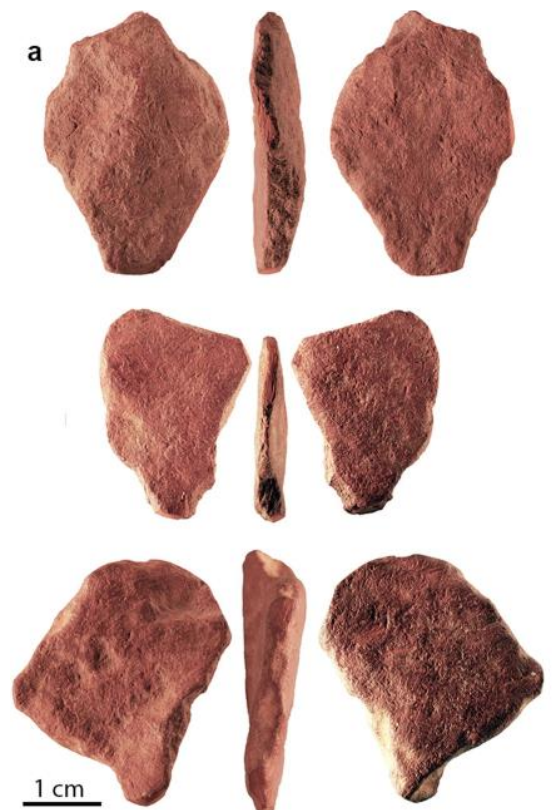
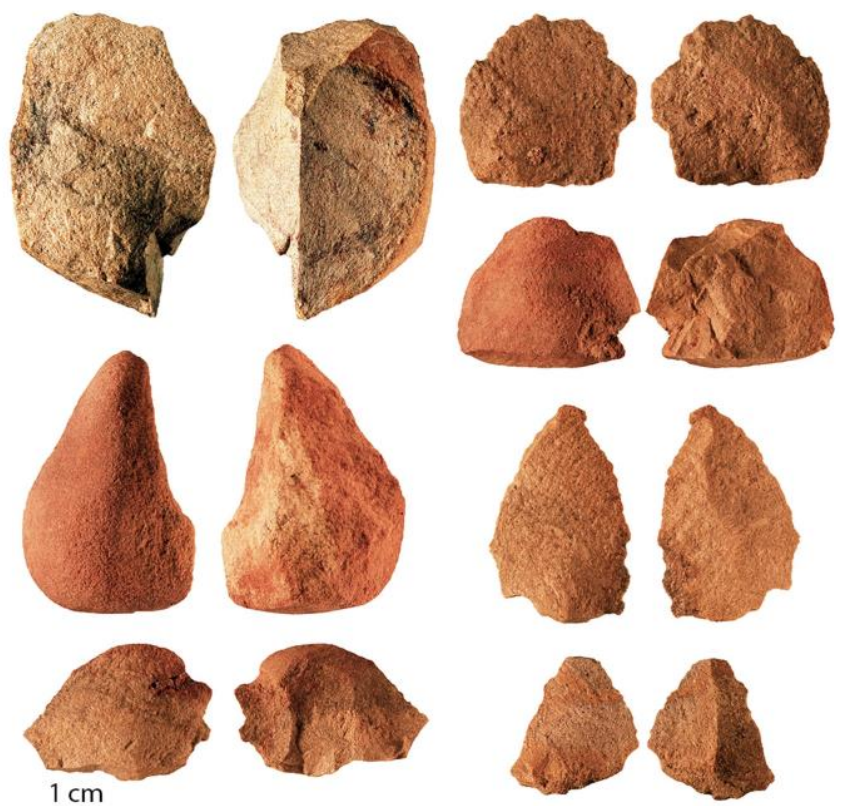

Extended Data Fig. 7. Ochre and silcrete used in the replication experiments. a, Pieces of ochre used experimentally to produce lines on silcrete flakes. $\mathbf{b}$, Silcrete flakes used during the experiments. 


\section{An abstract drawing from the 73,000 year old levels}

Page 2

- Microscopic examination and chemical analyses

Page 4

- Experimental marking of silcrete flakes

Page 5

- Ochre deposits from hammer-stone contact

Page 6

- References

Page 7

Supplementary Tables

- $\quad$ SI Table 1

Page 8

- $\quad$ SI Table 2

Page 8

- $\quad$ SI Table 3
Page 9 


\section{Microscopic examination and chemical analyses}

25 The red lines on L13 consist of juxtaposed patches of red deposits. The deposits accumulated in

26 micro-concavities are composed of fine-grained bright red particles. Deposits on flat areas form a thin

27 compact layer and are darker in colour. The latter are frequently associated with parallel striations

28 oriented along the direction of the line (Main text Fig. 4).

29 The red material consists mainly of fine grained iron oxide (Fe) (Extended data Fig. 1a-b, SI Table 2).

30 Variable contents of Si, associated with Fe, may come from the silcrete beneath the patches. Traces of

$31 \mathrm{Ti}$ in line 5 are probably part of the iron-rich material of this line. Ti is seldom detected in the silcrete

32 of L13 but, when present, does not show the same systematic association recorded on the red patches.

33 The two small red deposits on the flake scar adjacent to the marked surface also consist mainly of Fe

34 (SI Table 2). Haematite is identified by Raman within the red line deposits analysed (Extended data

35 Fig. 1c). Quartz is sometimes present but, as the red deposits are thin, the detected quartz may be a

36 component of the silcrete rather than the red patches. Iron-rich deposits are only associated with the

37 patches composing the lines, indicating that there is no haematite in the L13 silcrete.

38 The surface of the silcrete with the drawing is smoothed (see below, Tribological Analysis). The

39 quartz grains are flattened and some of the larger grains show micro-pitting associated, in some cases,

40 with incipient fractures (Extended data Fig. 2a). A few areas show microstriations that cross both the

41 matrix and the quartz grain. Micro-residues of red powder occasionally occur within depressions in

42 the matrix and the micro-pits. These residues are mainly composed of particles rich in iron oxide (Fe)

43 (Extended Data Fig. 2, SI Table 2). Haematite has been identified in all micro-residues analysed

44 (Extended data Fig. 2c). The significant concentration of Si detected by SEM-EDS and the quartz

45 signal on the Raman spectra are likely due to the underlying quartz grains. The Fe content of the

46 micro-residues is substantially higher than that of the natural silcrete, but lower than in the red lines

47 (see SI Table 2).

48 Silcrete is generally composed of quartz grains surrounded by a matrix of micro- to crypto-crystalline

49 silica with the possible presence of other minerals ${ }^{1,2}$. On the drawn face of L13 quartz grains are

50 relatively large and densely distributed within the matrix. Most are translucent but several are dark, 
51 often black in colour. SEM-EDS and Raman analyses confirm that these dark grains are quartz.

52 Grains of zircon (Zr, Si) are also identified. The matrix of L13 comprises silica (Si), possible clay

53 minerals ( $\mathrm{Si}, \mathrm{Al}$ ), calcite (Ca, C), and iron oxide (Fe) (SI Table 2). Some of these elements may derive

54 from the sediment in which L13 was deposited. The carbon detected on the surface could be from a

55 thin calcite layer or indicate an organic coating.

56 To clarify the composition of the silcrete and the taphonomic history of L13, further analyses were

57 done on the dorsal surface of L13, opposite to the drawn surface. This face is composed of silica

58 grains ( $\mathrm{Si}$ ) and the matrix is rich in $\mathrm{Si}$ (SI Table 2). As Ca and $\mathrm{C}$ are the two most dominant elements

59 after $\mathrm{Si}$, it is likely that a thin layer of calcite is present on this surface, similar to that seen on the

60 drawn surface. Significantly, the Fe concentration is very low on this face compared to that in the red

61 lines and the micro-residues in-between the quartz grains on the drawn surface (SI Table 2). Orange

62 spots, visible under the microscope, are not detected with SEM back-scattered electron imagery

63 indicating the absence of heavy elements such as Fe. There is an unusual presence of $\mathrm{Ti}$ and Fe in one

64 of the spots analysed (SI Table 2), which may derive from the surrounding sediment.

65 The chemical composition and microscopic analysis of the red lines on L13, indicates that ochre was

66 applied to the surface. Experimental reproduction of these lines on silcrete flakes using two

67 techniques (See above, Methods, SI Table 1 and Extended data figs. 3) indicate that they were

68 produced by marking the surface with the edge of a thin ochre piece. The microscopic deposits of iron

69 oxide rich particles detected on the drawn surface outside of the lines, and found only on this surface,

70 are anthropogenic. They are mainly located on the smoothest areas, and are associated with micro-pits

71 on quartz grains and striations identified by confocal microscopy. They are likely due to grinding

72 ochre on this surface prior to the application of the red lines. The faint nature of these deposits

73 suggests that the surface was cleaned, removing most of the ochre, before the lines were drawn. The

74 red deposit on the flake scar, adjacent to the smoothed surface of L13, may result from unsuccessful

75 attempts to produce a line on this rough surface.

76 Elemental composition of the different items analysed: the particles composing the red powder of the

77 lines; the particles of the red micro-agglomerates; the grains and the matrix of the silcrete. Semi- 
78 quantitative data have been used to range each element (weight percentages normalized to $100 \%$, with

79 C and O included).

\section{Experimental marking of silcrete flakes}

81 Tracing a line of red paint with a thin wooden brush on a flat silcrete surface produces an elongated 82 stripe entirely covered by paint with well-defined edges (Extended Data Fig. 3a). The application of a 83 thin paint results in a homogenous layer (Extended Data Fig. 3a[a and d]) whereas with medium

84 density paint the edges of the line become more irregular, the mixture accumulates in concave areas

85 and displays cracks after drying (Extended Data Fig. 3a[b and e]). Thick paint produces a

86 discontinuous line with accumulations of paint in places along the line edges (Extended Data Fig. 3a[c

87 and f]). As with the medium density paint, numerous cracks appear after drying.

88 In all three cases, although rinsing under running water removes the accumulations of dried paint, the

89 painted surface remains homogeneously covered by ochre residues and the painted and non-painted

90 surfaces remain clearly distinguishable (Extended Data Fig. 3a[g-i]). With thick paint, the edges of the

91 line are marked by a thin continuous boundary (Extended Data Fig. 3a[i]).

92 Lines made by a single stroke of an ochre crayon (with a pointed or linear edge) on a silcrete surface

93 result in discontinuous patches of ochre with irregular outlines (Extended Data Fig. 3b, SI Table 1).

94 In some patches there are two distinct areas: a recess, partially filled with ochre powder, followed by a

95 flat area covered by a deposit of ochre with parallel striations (Extended Data Fig. 3b[b and c]). The

96 relative location of these two areas indicates the direction of the movement of the ochre crayon while

97 producing the line. The shaving effect of micro-protrusions on the silcrete produces powder that

98 accumulates in recesses. The pressure then produces a compacted streak of ochre on the following

99 raised surface. Striations on this coating are produced by abrasive particles present in the ochre. One-

100 or multiple- strokes only leaves a minute amount of loose ochre powder on the silcrete flake.

101 Cleaning the silcrete flakes marked with a crayon by rinsing with water removes the excess loose

102 powder, leaving the compacted ochre lines in situ (Extended Data Fig. 3b [h and i]). Striations on the

103 lines are still visible. Superimposing a line on a previous line generally results in a wider line as it is

104 difficult to exactly superimpose a new line on the previous one. Unidirectional, superimposed lines 
retain the same features observed on a single stroke line. Multiple lines produced by a to-and-fro movement of the ochre edge show microscopic evidence that the crayon was moved in both directions (Extended Data Fig. 4).

Experimental marking of silcrete flakes with a variety of ochre crayons demonstrates that the morphology of lines will depend on the properties and composition of the ochre, the roughness of the silcrete surface, the pressure exerted, and the morphology of the ochre area in contact with the silcrete. In general, soft, plastic, clayish ochre will produce thicker, more continuous lines than silty or sand-rich ochre. Lines on fine grained silcrete will be better defined than on coarse silcrete. Stronger pressure will produce comparatively wider, thicker and better defined lines. Six lines made with each of eight unmodified ochre crayons had a maximum width ranging from c. 0.9 to $3.3 \mathrm{~mm}$. When produced with a pointed ochre crayon the lines tend to be wider and more variable in width than those made with a linear edge (Extended Data fig. 5). The width of the latter is strongly correlated to the maximum width of the facets on the ochre piece. In contrast, no correlation is observed between the lines made with pointed crayons and the maximum width of the facets on the crayon. The width of the lines on the drawn cross-hatching present on L13 is comparable with that of the experimental lines. Its range $(1.8-2.9 \mathrm{~mm})$ best fits the width variability observed when marking the silcrete with a pointed crayon rather than an edge. It indicates that a pointed ochre crayon was used to produce the cross hatching and that the facet of the crayon in contact with the silcrete was c. 1.3-2.9 mm wide.

\section{Ochre deposits on striking platforms resulting from hammer-stone contact}

We considered the possibility that the L13 drawing could result from ochre deposits left by an ochre or ochre-laden hammer-stone when the L13 flake was struck. Soriano et al. ${ }^{3}$ record ochre residues on struck flakes recovered from the Still Bay (c.71 ka) levels at Sibudu Shelter. In their Plate 1 (pp.54) these flakes are clearly illustrated and the ochre splodges on the struck surfaces bear no resemblance to the pattern on L13. Soriano et al. ${ }^{3}$ consider several ways in which ochre could have been deposited on the Sibudu flakes and favour the hypothesis of a direct utilization of ochre nodules as hammers. During our ongoing but unpublished lithic analysis of flakes from the Still Bay levels at Blombos Cave (c. 75-72 ka) we also note ochre traces on some platforms resembling those illustrated from 
132 Sibudu. None of the ochre traces on these Blombos flakes bear any resemblance to the deliberate

133 pattern on L13. As is the case for Sibudu, we have not recovered ochre hammer-stones in the Still

134 Bay levels at Blombos Cave.

135 Even if ochre hammer-stones were used at Sibudu or Blombos Cave in the Still Bay levels there can

136 be no relationship between these hammer-stones and the ochre crayon used to draw the design on

137 L13. The thickness of the lines on L13 is indicative of a thin and light ochre piece that is incompatible

138 with being used as a hammer. Full support for this hypothesis is provided earlier in this paper where

139 we describe, experimentally, how we reproduce these lines and how we are thus certain of the method

140 of application and the size of the crayon - the hammer-stone application method is thus a null

141 hypothesis.

\section{Tribological analysis}

143 3D rendering of microscopic areas of L13 and silcrete flakes from BBC identifies flattening of the

144 drawn surface of L13 (Extended Data Fig. 6a[a]), dissolution of the matrix between quartz grains on

145 the cortex of the BBC silcrete flakes (Extended Data Fig.6a[d]) and an unworn appearance of the

146 other surfaces of L13 (Extended Data Fig. 6a[b]) and the ventral aspect of the BBC silcrete flakes

147 (Extended Data Fig. 6a[c]). The latter two display well-preserved quartz grains featuring typical

148 conchoidal fractures and no traces of cement dissolution. Three roughness parameters were able to

149 distinguish the four groups of surfaces: $S q$ (root-mean-square height), $S d r$ (developed interfacial area

150 ratio), which respectively measure the overall roughness of the surface and its complexity, and Spc,

151 which measures the mean form of the peaks (pointed $v$ s rounded). Results indicate that the drawn

152 surface of L13 is smoother than the other surfaces of this piece and the ventral and cortical surfaces of

153 the BBC flakes (Extended Data Fig. 6a[e]). Higher Sq, Sdr, and $S p c$ values, indicating increased

154 surface roughness, were measured on cortical surfaces. Such high values are due to weathering which

155 dissolves the matrix, thus exposing the quartz grains. A Kruskall-Wallis multiple comparison test

156 demonstrates that $S q, S d r$ and $S p c$ on the drawn surface is significantly lower $(\mathrm{p}<0.01)$ than that

157 measured on the remainder of the analysed surfaces of L13 (SI Table 3). 
158 Areal fractal analysis confirms a clear difference in roughness between the drawn and other surfaces

159 of L13, which is consistent with the interpretation of the wear on the former as produced by grinding

160 activities prior to the drawing (Extended data Fig. 6b). It also reveals that the dorsal (Extended data

161 Fig 6c[d]) and ventral (Extended data Fig. 6c[e]) surfaces of the flake are rougher than the flake scars

162 on the drawn (Extended data Fig. 6c[b]) and ventral face (Extended data Fig. 6c[c]). This suggests

163 that these surfaces had slightly different taphonomic histories.

164 References

16531 Blatt, H., Tracy, R. J. \& Owens, B. Petrology-Igneous. Sedimentary, and Metamorphic: WH 166 Freeman \&Co, New York, 377-380 (1996).

16732 Nash, D. J. \& Ullyott, J. S. in Geochemical sediments and landscapes Vol. Nash, D.J. \& Ullyott, 168 J.S., 2007. Silcrete, in . (eds) Geochemical Sediments \& Landscapes., pp.95-143. (eds D.J. $169 \quad$ Nash \& S.J. McLaren) 95-148 (Wiley-Blackwell, 2007).

170

33 Soriano, S., Villa, P. \& Wadley, L. Ochre for the toolmaker: shaping the Still Bay points at Sibudu (KwaZulu-Natal, South Africa). Journal of African Archaeology 7, 41-54 (2009).

172

173

174

175

176

177

178

179

180

181

182

183

184 
186 SI Table 1 | Data on experimental ochre crayons and resulting lines.

\begin{tabular}{|c|c|c|c|c|c|c|c|c|c|c|c|c|c|}
\hline Tip & $\begin{array}{l}\text { active area } \\
\text { morphology }\end{array}$ & $\begin{array}{c}\text { lithic surface } \\
\text { type }\end{array}$ & orientation of the tip & $\begin{array}{c}\text { Crayon } \\
\text { tip angle } \\
\left({ }^{\circ}\right)\end{array}$ & $\begin{array}{l}\text { Width at } 3 \\
\mathrm{~mm} \text { from the } \\
\text { tip (mm) }\end{array}$ & $\begin{array}{l}\text { Thickness } \\
\text { at } 3 \mathrm{~mm} \\
\text { from the tip } \\
(\mathrm{mm})\end{array}$ & $\begin{array}{l}\text { Number } \\
\text { of lines }\end{array}$ & $\begin{array}{l}\text { Mean } \\
\text { length of } \\
\text { the lines } \\
(\mathrm{mm})\end{array}$ & $\begin{array}{l}\text { Fracture } \\
\text { of the } \\
\text { crayon }\end{array}$ & $\begin{array}{l}\text { Min width } \\
\text { mean } \\
(\mathrm{mm})\end{array}$ & SD & $\begin{array}{l}\text { Max } \\
\text { width } \\
\text { mean } \\
(\mathrm{mm})\end{array}$ & SD \\
\hline 1 & pointed & debitage & parallel to the width & 92 & 6.43 & 3.81 & 6 & 41.1 & no & 2,083 & 0,648 & 1,445 & 0,786 \\
\hline 2 & pointed & debitage & parallel to the width & 71 & 6.72 & 6.01 & 6 & 45.5 & no & 1,908 & 0,077 & 1,178 & 0,091 \\
\hline 3 & pointed & debitage & perpendicular to the width & 75 & 6.78 & 3.36 & 6 & 44.5 & no & 2,003 & 0,18 & 1,203 & 0,313 \\
\hline 4 & pointed & cortex & perpendicular to the width & 75 & 7.45 & 2.98 & 6 & 45.5 & no & 2,632 & 0,627 & 1,343 & 0,192 \\
\hline 5 & linear & debitage & parallel to the width & 145 & 10.53 & 2.25 & 6 & 34 & no & 1,167 & 0,139 & 0,948 & 0,06 \\
\hline 6 & linear & debitage & parallel to the width & 170 & 17.68 & 3.13 & 6 & 39.5 & no & 1,725 & 0,215 & 1,325 & 0,297 \\
\hline 7 & linear & debitage & parallel to the width & 180 & 13.33 & 2.93 & 6 & 44.3 & no & 1,828 & 0,283 & 1,457 & 0,133 \\
\hline 8 & linear & debitage & parallel to the width & 160 & 9.15 & 3.71 & 6 & 40.2 & no & 1,985 & 0,338 & 1,282 & 0,209 \\
\hline
\end{tabular}

SI Table 2 | Summary of the SEM-EDS analyses on the lines and other surfaces of L13.

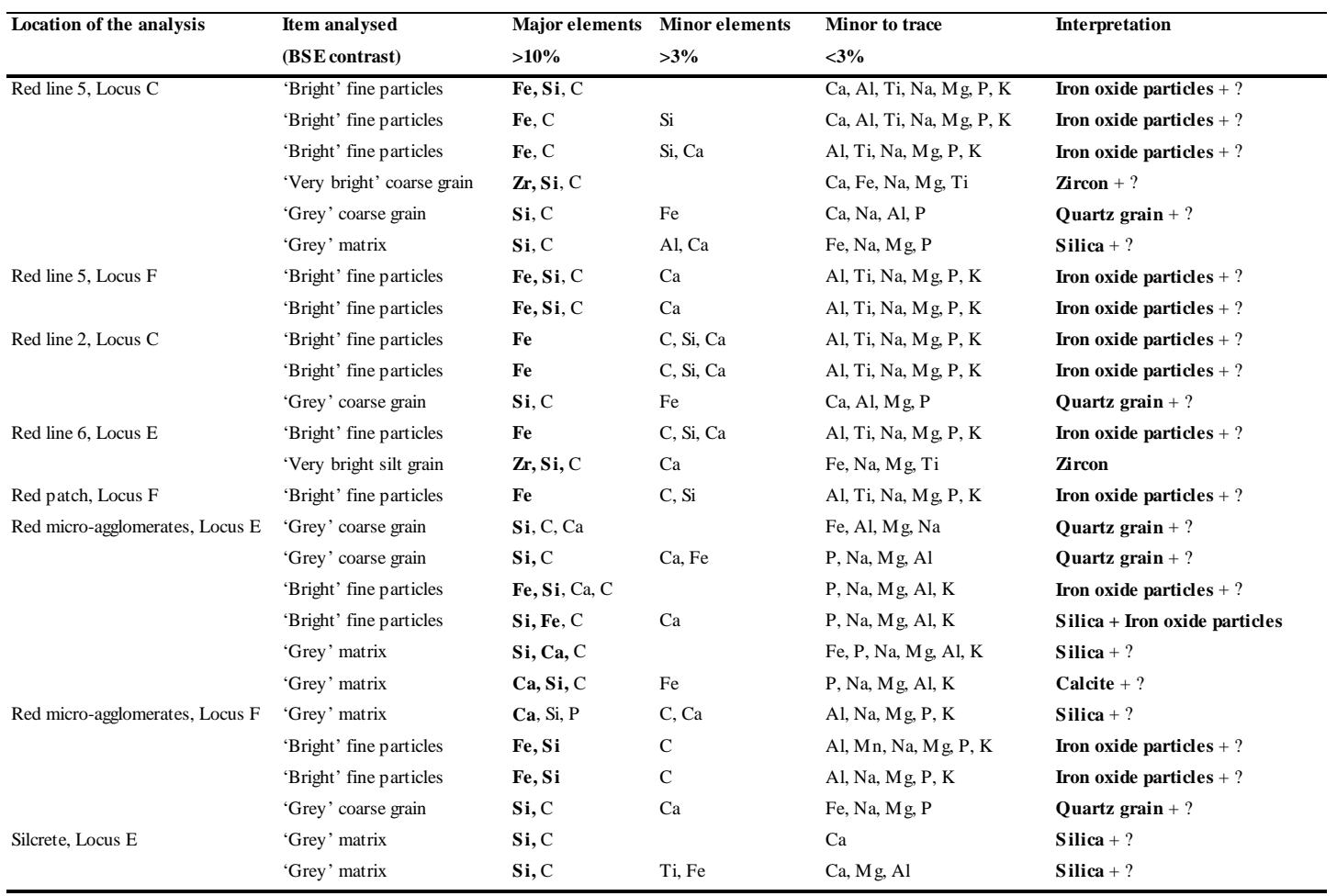

191 


\begin{tabular}{|c|c|c|}
\hline Kruskall-Wallis $(S q)$ & \multicolumn{2}{|c|}{$p=5.946458 \mathrm{e}-23$} \\
\hline Comparison between groups & $p<0,05$ & $p<0,01$ \\
\hline BBC L13 - drawn surface vs. BBC L13 - other surfaces & TRUE & TRUE \\
\hline BBC L13 - drawn surface vs. BBC MSA flakes - knapped surfaces & TRUE & TRUE \\
\hline BBC L13 - drawn surface vs. BBC MSA flakes - cortical surfaces & TRUE & TRUE \\
\hline BBC L13 - other surfaces vs. BBC MSA flakes - knapped surfaces & TRUE & FALSE \\
\hline BBC L13 - other surfaces vs. BBC MSA flakes - cortical surfaces & TRUE & TRUE \\
\hline BBC MSA flakes - knapped surfaces vs. BBC MSA flakes - cortical surfaces & TRUE & TRUE \\
\hline Kruskall-Wallis (Sdr) & \multicolumn{2}{|c|}{$p=3.207647 \mathrm{e}-22$} \\
\hline Comparison between groups & $p<0,05$ & $\mathrm{p}<0,01$ \\
\hline BBC L13 - drawn surface vs. BBCL13 - other surfaces & TRUE & TRUE \\
\hline BBC L13 - drawn surface vs. BBC MSA flakes - knapped surfaces & FALSE & FALSE \\
\hline BBC L13 - drawn surface vs. BBC MSA flakes - cortical surfaces & TRUE & TRUE \\
\hline BBC L13 - other surfaces vs. BBC MSA flakes - knapped surfaces & TRUE & TRUE \\
\hline BBC L13 - other surfaces vs. BBC MSA flakes - cortical surfaces & TRUE & TRUE \\
\hline BBC MSA flakes - knapped surfaces vs. BBC MSA flakes - cortical surfaces & TRUE & TRUE \\
\hline Kruskall-Wallis $(S p c)$ & $p=3.74$ & \\
\hline Comparison between groups & $\mathrm{p}<0,05$ & $\mathrm{p}<0,01$ \\
\hline BBC L13 - drawn surface vs. BBCL13 - other surfaces & TRUE & TRUE \\
\hline BBC L13 - drawn surface vs. BBC MSA flakes - knapped surfaces & FALSE & FALSE \\
\hline BBC L13 - drawn surface vs. BBC MSA flakes - cortical surfaces & TRUE & TRUE \\
\hline BBC L13 - other surfaces vs. BBC MSA flakes - knapped surfaces & TRUE & TRUE \\
\hline BBC L13 - other surfaces vs. BBC MSA flakes - cortical surfaces & FALSE & FALSE \\
\hline BBC MSA flakes - knapped surfaces vs. BBC MSA flakes - cortical surfaces & TRUE & TRUE \\
\hline
\end{tabular}

